\title{
Angioscotomata and morphological features of related vessels in automated perimetry
}

\author{
A B Safran, A Halfon, E Safran, C Mermoud
}

\begin{abstract}
Aims-To determine principles which regulate the occurrence of angioscotomata in automated static perimetry, variations in light sensitivity were correlated with the location and diameter of neighbouring retinal vessels.
\end{abstract}

Methods-Ten normal eyes were tested with the Octopus $2000 \mathrm{R}$, using a $0.431^{\circ}$ light stimulus. Sensitivity was quantified in points located around the blind spot, according to a regular, $0 \cdot 5^{\circ}$ constant, grid pattern. From 336 to 443 locations were tested in each eye. The resulting printouts were superimposed on corresponding fundus photographs. At each tested point, the following five additional variables were evaluated: the diameters of the closest and the second closest vessel (in $0.1^{\circ}$ units); the distances of the apparent location of the tested point to the closest and the second closest vessel (in $0.25^{\circ}$ units); and the distance between the two closest vessels (in $0 \cdot 25^{\circ}$ units). Altogether, 3869 locations were tested and 23214 values were quantified.

Results-The following two conditions were found to be related to a reduction in sensitivity: (1) proximity $\left(<0.25^{\circ}\right)$ to a large vessel ( $\geqslant 0.5^{\circ}$ in diameter); (2) proximity $\left(<0 \cdot 25^{\circ}\right)$ to one of two adjacent $\left(<0.5^{\circ}\right.$ distant $)$, moderately large vessels $\left(0.3^{\circ}\right.$ to $0.4^{\circ}$ in diameter). In condition 1 , sensitivity was $51.3 \%$ and specificity was $92 \cdot 2 \%$; in condition 2 , sensitivity was $16 \cdot 2 \%$ and specificity was $98.3 \%$; and with a combination of conditions 1 and 2 , sensitivity was $67.6 \%$ and specificity was $90.5 \%$. Increase by $0.1^{\circ}$ of an adjacent vessel which was $0.4^{\circ}$ in diameter markedly affected light sensitivity.

Conclusion-Modifications in vessel diameter are observed in a number of circumstances, including adaptive vascular response to changes in ambient conditions and obstructive disorders of retinal vessels. These findings indicate that changes in vessel diameter over time can result in fluctuation of sensitivity. It is concluded that, in contrast with what is commonly stated, when ocular media are unaltered and the subject's collaboration is adequate, temporal variations in measured thresholds do not necessarily reflect functional changes in nervous tissues in the visual pathways.

(Br F Ophthalmol 1995; 79: 118-124)

One of the most crucial problems when dealing with automated static perimetry is determining the criteria of normality for the measured values, particularly with regard to spatial and temporal variations. Variation in light sensitivity across the retina is due partly to the presence of vessels at the surface of the retina, which prevent light from reaching some areas of the retina, thus causing so-called angioscotomata. ${ }^{1}$

Several decades ago, angioscotomata were extensively studied by manual perimetry, mainly of the kinetic type. ${ }^{1}$ With automated perimetry, however, little attention has been paid to angioscotomata. ${ }^{2-6}$ This results from the fact that the stimulus locations generally used in automatic static perimetry for examination of the central field are based upon a $6^{\circ}$ interstimulus separation. The resolution of such programs is not appropriate for the detection and spatial delineation of tiny localised scotomata. This is due mainly to the need to limit the number of tested locations, in order to keep the examination time to a duration which is acceptable to the tested subject and yields reliable results.

Even if angioscotomata are not well delineated by routine strategies used in automated perimetry, knowledge of their characteristics is essential for understanding how the retinal vessels influence values regularly obtained with this technique.

We studied how the morphological characteristics of retinal vessels affected the light sensitivity measured in corresponding areas of the visual field. Our technique was based on superimposing the data obtained by automated static perimetry on photographs of the fundus.

\section{Materials and methods}

Six normal subjects (aged 23 to 29 years) were involved in the study. Inclusion criteria were as follows: (1) uncorrected visual acuity of at least 20/20; (2) no history of ocular disease; and (3) no abnormality on ophthalmic examination. Both eyes were examined in the first four subjects, but only the right eye was evaluated in the other two.

The study was conducted with a perimetric examination program using a personal computer connected to the measuring unit of an Octopus 2000R automated perimeter. The evaluation procedure was as follows. Firstly, in each tested eye the centre of the blind spot was located using a spatially adaptive strategy, which we have described elsewhere. ${ }^{7}$ The differential light threshold was then fully quantified at points located within an area centred on the blind spot, using the highest spatial resolution of the instrument - that is, a $0.5^{\circ}$ constant grid pattern of tested points. The blind spot was defined as the surface which 


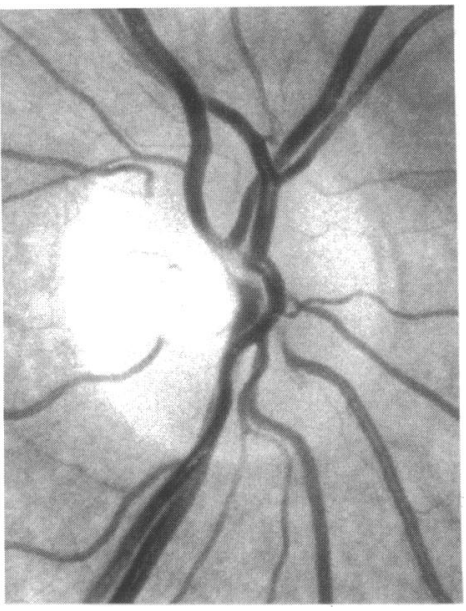

Fig $1 A$

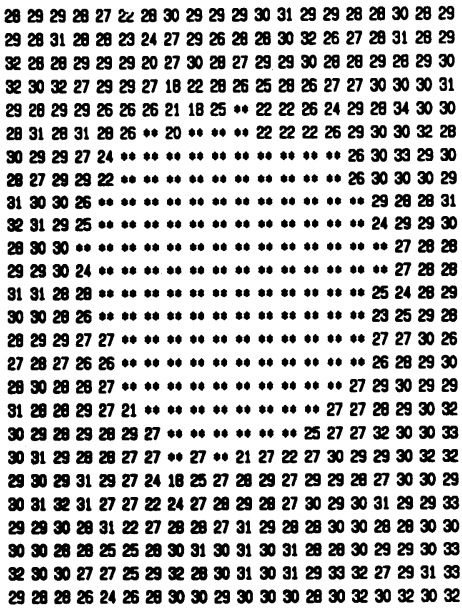

Fig $1 B$
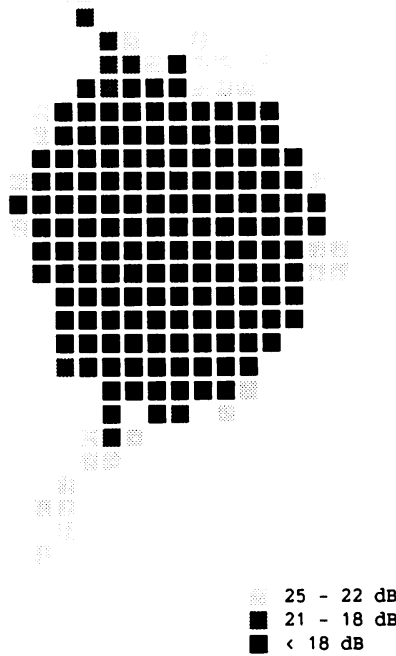

Fig $1 C$

Figure 1 Fundus appearance (A), actual results of the visual field evaluation (B), and grey scale printout (C) in the examined area in the right eye of subject 2 . The fundus picture is upside down, to allow comparison with the printout. Tested points are located on a $0.5^{\circ}$ in constant grid pattern. ${ }^{\star}$ Indicates that the tested location is less than $18 \mathrm{~dB}$ in sensitivity.

includes all tested locations showing a light sensitivity of $17 \mathrm{~dB}$ or less, plus one row of tested points surrounding this surface. The examined area varied from $8.0^{\circ}$ to $9.5^{\circ}$ in width, and from $12.5^{\circ}$ to $15^{\circ}$ in height. Because of its long duration, the examination was performed by successively testing small adjacent surfaces of the visual field, each including about 50 tested locations. The subjects were allowed to rest between each examination period. The results were printed out both as numerical values and in grey scales, in a pattern corresponding to the tested locations.

Later, after pupil dilatation, photographs were taken of the fundus of each tested eye, using a Canon CF 60 UV fundus camera. Photographs were $30^{\circ}$ in angle and centred on the fovea. Paper prints of the photographs were magnified to match the printouts resulting from the visual field evaluation. Transparencies were made of the inverted printouts and were superimposed on the fundus photographs, matching the centre of the blind spot to that of the optic disc, and the fixation point of the visual field to the foveola (Figs 1, 2). In addition, based on the magnification of the visual field printout, and with the use of a computer, a scale was printed in $0.1^{\circ}$ steps, and used to measure angular distances on the photographs.

For each tested point, the corresponding location on the retina was determined, and the two closest vessels larger than $0.05^{\circ}$ in diameter were identified and classified as closest vessel (vessel 1) or second closest vessel (vessel 2). The following five parameters were then evaluated for each tested point: the diameters of vessels 1 and 2 (in $0 \cdot 1^{\circ}$ units); the distances of the tested point from vessels 1 and

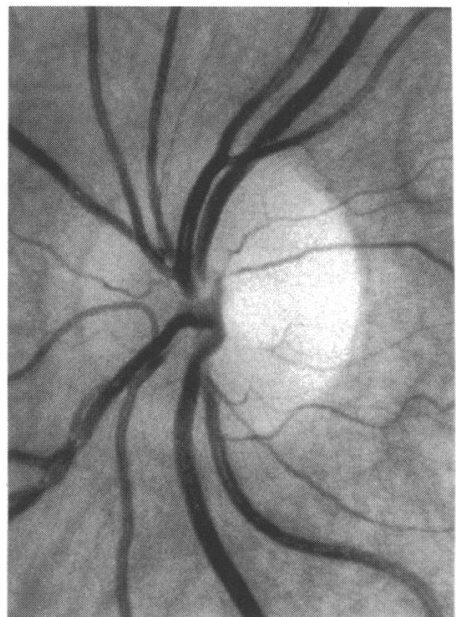

Fig $2 A$

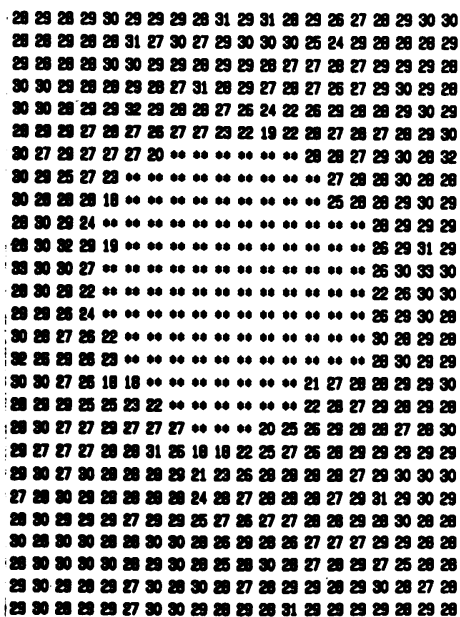

Fig $2 B$

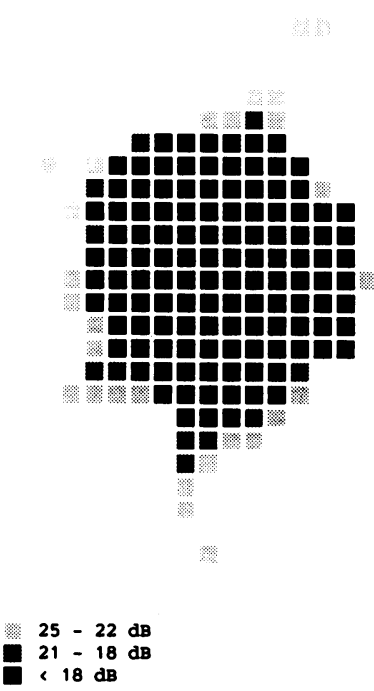

Fig $2 C$

Figure 2 Data obtained from the left eye of subject 2. When comparing this figure with that of the right eye of the same subject (Fig 1), it appears that (1) in both eyes the main retinal vessels are similar in size - that is, $0 \cdot 3^{\circ}$ to $0 \cdot 4^{\circ}$ in diameter; (2) these vessels occur in pairs, consisting of an arteriole and a venule, which are generally closer together in the right eye than in the left eye; and (3) in the visual field areas corresponding to these vessels, the number of low sensitivity points ( $L O S$ points) is greater in the right eye than in the left eye. 


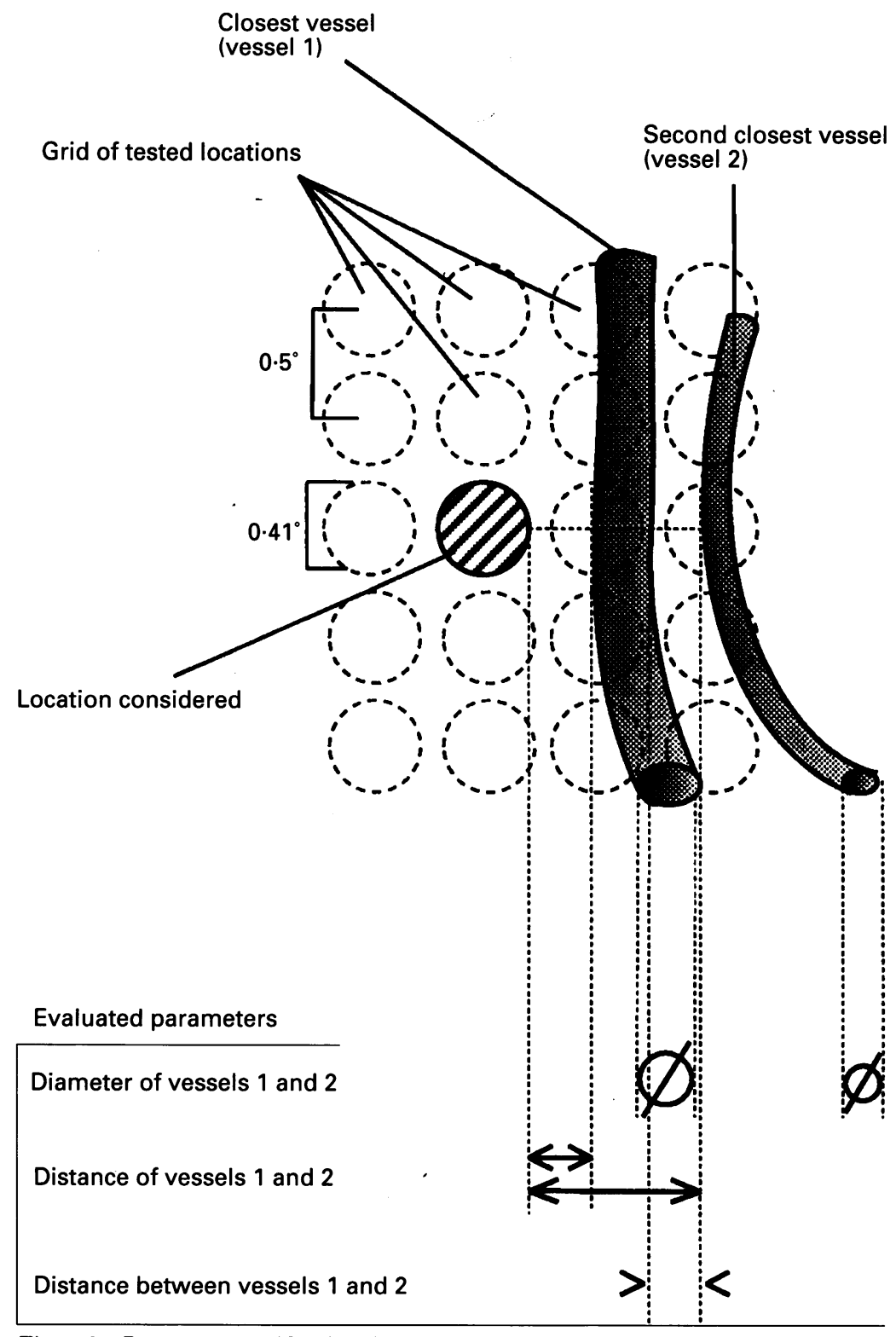

Figure 3 Parameters considered in the study.

2 (in $0.25^{\circ}$ units); and the distance between vessels 1 and 2 (in $0.25^{\circ}$ units) (Fig 3).

Values of the vessel diameters were rounded up to the nearest tenth of a degree. Distances of the tested point from the vessels were rounded up to the nearest quarter of a degree, and were categorised as $1.25^{\circ}$ or more distant, $1.0^{\circ}$ distant, $0.75^{\circ}$ distant, $0.5^{\circ}$ distant, $0.25^{\circ}$ distant, adjacent to the vessel, overlapping the vessel, or projected onto the centre of the vessel. Distances between vessels 1 and 2 were categorised as $1.25^{\circ}$ or more apart, $1.0^{\circ}$ apart, $0.75^{\circ}$ apart, $0.5^{\circ}$ apart, $0.25^{\circ}$ apart, adjacent vessels, or crossing vessels.

Data obtained from all tested eyes were pooled, and reference values for sensitivity were calculated using the values obtained at tested points which were $1.25^{\circ}$ or more distant from any considered vessel - that is, in locations presumed to be free of angioscotomata. The 0.5 percentile of sensitivity values at these points was determined. Next, all points showing a sensitivity below this limit were defined as low sensitivity points (LoS points); their variables were compared with those of the remaining locations. Morphological features of the retinal vessels that were related to these $\operatorname{LoS}$ points were identified; this association was evaluated statistically in terms of sensitivity, specificity, and positive and negative predictive values. Subsequently, each eye was evaluated separately.

\section{Results}

Excluding points defined as located within the surface of the blind spot, the total number of tested locations was $3869 ; 336$ to 443 locations were assessed in each eye (Table 1). We measured a total of 23214 values of the various parameters.

In the 507 locations which were $1 \cdot 25^{\circ}$ or more distant from the closest considered vessel, mean light sensitivity was $30.2 \mathrm{~dB} ; 11$ locations $(2 \cdot 2 \%)$ were less than $27 \mathrm{~dB}$ in sensitivity; and only two $(0.4 \%)$ were less than 26 $\mathrm{dB}$. Therefore, we subsequently considered that values below $26 \mathrm{~dB}$ showed a significant reduction in sensitivity, and corresponding locations were called low sensitivity points (LoS points).

Of the 3869 tested locations, 148 were LoS points. The evaluated parameters related to these 148 points were studied, and compared with those of the remaining 3721 locations.

\section{INFLUENCE OF THE DIAMETERS OF VESSELS 1} AND 2

Firstly, the proportion of LoS points was analysed with regard to the diameter of vessel 1

Table 1 Evaluation of positive predictive value (PPV), negative predictive value (NPV), sensitivity (Sens), and specificity (Spec) of the conditions found to be related to a reduction in sensitivity. Conditions were considered separately and in combination. Analysis was performed for individual field examinations and with data pooled from all tested fields

\begin{tabular}{|c|c|c|c|c|c|c|c|c|c|c|c|c|c|c|c|c|c|}
\hline \multirow[b]{2}{*}{$\begin{array}{l}\text { Subj, } \\
\text { eye }\end{array}$} & \multirow[b]{2}{*}{$\begin{array}{l}\text { Tested } \\
\text { pts }\end{array}$} & \multirow[b]{2}{*}{$\operatorname{LoS} p t s$} & \multicolumn{5}{|c|}{ Condition 1} & \multicolumn{5}{|c|}{ Condition 2} & \multicolumn{5}{|c|}{ Condition 1 or 2} \\
\hline & & & $\begin{array}{l}\text { Tested } \\
\text { pts }\end{array}$ & $P P V$ & $N P V$ & Sens & Spec & $\begin{array}{l}\text { Tested } \\
\text { pts }\end{array}$ & $P P V$ & $N P V$ & Sens & Spec & $\begin{array}{l}\text { Tested } \\
\text { pts }\end{array}$ & $P P V$ & $N P V$ & Sens & Spec \\
\hline $\begin{array}{l}\text { 1, RE } \\
\text { 1, LE } \\
\text { 2, RE } \\
\text { 2, LE } \\
3, \mathrm{RE} \\
3, \mathrm{LE} \\
\text { 4, RE } \\
\text { 4, LE } \\
\text { 5, RE } \\
6, \mathrm{RE} \\
\text { Total }\end{array}$ & $\begin{array}{l}433 \\
341 \\
353 \\
360 \\
407 \\
385 \\
380 \\
336 \\
431 \\
443 \\
3869\end{array}$ & $\begin{array}{r}13(3.0 \%) \\
11(3.2 \%) \\
23(6.5 \%) \\
12(3.3 \%) \\
16(3.9 \%) \\
15(3.9 \%) \\
15(3.9 \%) \\
13(3.9 \%) \\
9(2.1 \%) \\
21(4.7 \%) \\
148(3.8 \%)\end{array}$ & $\begin{array}{r}50 \\
48 \\
16 \\
0 \\
57 \\
34 \\
18 \\
39 \\
52 \\
53 \\
367\end{array}$ & $\begin{array}{l}12 \cdot 0 \\
14 \cdot 6 \\
50 \cdot 0 \\
- \\
21 \cdot 0 \\
29 \cdot 4 \\
38 \cdot 9 \\
23 \cdot 1 \\
11 \cdot 5 \\
20 \cdot 8 \\
20 \cdot 7\end{array}$ & $\begin{array}{l}98 \cdot 2 \\
98 \cdot 6 \\
95 \cdot 6 \\
96 \cdot 6 \\
98 \cdot 9 \\
98 \cdot 6 \\
97 \cdot 8 \\
98 \cdot 7 \\
99 \cdot 2 \\
97 \cdot 4 \\
97 \cdot 9\end{array}$ & $\begin{array}{c}46 \cdot 2 \\
63 \cdot 6 \\
34 \cdot 8 \\
0 \\
75 \\
66 \cdot 7 \\
46 \cdot 7 \\
69 \cdot 2 \\
66 \cdot 7 \\
52 \cdot 4 \\
51 \cdot 3\end{array}$ & $\begin{array}{c}89 \cdot 5 \\
87 \cdot 6 \\
97 \cdot 6 \\
100 \\
88 \cdot 5 \\
93 \cdot 5 \\
97 \cdot 0 \\
90 \cdot 7 \\
89 \cdot 1 \\
90 \cdot 1 \\
92 \cdot 2\end{array}$ & $\begin{array}{r}21 \\
21 \\
37 \\
17 \\
3 \\
21 \\
20 \\
0 \\
18 \\
24 \\
182\end{array}$ & $\begin{array}{r}9 \cdot 5 \\
19 \cdot 1 \\
40 \cdot 5 \\
35 \cdot 3 \\
\overline{9} \\
9 \cdot 5 \\
20 \cdot 0 \\
\overline{5} \\
29 \cdot 6 \\
22 \cdot 5\end{array}$ & $\begin{array}{l}97 \cdot 3 \\
97 \cdot 8 \\
97 \cdot 6 \\
98 \cdot 3 \\
96 \cdot 0 \\
96 \cdot 4 \\
96 \cdot 9 \\
96 \cdot 1 \\
98 \cdot 1 \\
96 \cdot 7 \\
97 \cdot 1\end{array}$ & $\begin{array}{r}15 \cdot 4 \\
36 \cdot 4 \\
65 \cdot 2 \\
50 \cdot 0 \\
0 \cdot 0 \\
13 \cdot 3 \\
26 \cdot 7 \\
0 \cdot 0 \\
11 \cdot 1 \\
33 \cdot 3 \\
27 \cdot 7\end{array}$ & $\begin{array}{c}95 \cdot 5 \\
94 \cdot 8 \\
93 \cdot 3 \\
96 \cdot 8 \\
99 \cdot 2 \\
94 \cdot 9 \\
95 \cdot 6 \\
100 \\
96 \cdot 0 \\
96 \cdot 0 \\
96 \cdot 2\end{array}$ & $\begin{array}{r}69 \\
64 \\
48 \\
17 \\
60 \\
55 \\
36 \\
39 \\
69 \\
76 \\
533\end{array}$ & $\begin{array}{l}10 \cdot 1 \\
14 \cdot 1 \\
41 \cdot 8 \\
35 \cdot 3 \\
20 \cdot 0 \\
21 \cdot 8 \\
30 \cdot 6 \\
23 \cdot 8 \\
10 \cdot 1 \\
22 \cdot 4 \\
20 \cdot 6\end{array}$ & $\begin{array}{l}98 \cdot 3 \\
99 \cdot 3 \\
99 \cdot 0 \\
98 \cdot 3 \\
98 \cdot 8 \\
99 \cdot 1 \\
98 \cdot 8 \\
98 \cdot 6 \\
99 \cdot 5 \\
98 \cdot 9 \\
98 \cdot 9\end{array}$ & $\begin{array}{l}53 \cdot 8 \\
81 \cdot 8 \\
87 \cdot 0 \\
50 \cdot 0 \\
75 \cdot 0 \\
80 \cdot 0 \\
73 \cdot 3 \\
69 \cdot 2 \\
77 \cdot 8 \\
81 \cdot 0 \\
74 \cdot 3\end{array}$ & $\begin{array}{l}85 \cdot 2 \\
83 \cdot 3 \\
91 \cdot 5 \\
96 \cdot 8 \\
87 \cdot 7 \\
88 \cdot 4 \\
93 \cdot 1 \\
90 \cdot 7 \\
85 \cdot 3 \\
86 \cdot 0 \\
88 \cdot 6\end{array}$ \\
\hline
\end{tabular}

Subj=subjects; $R E=$ right eye; $\mathrm{LE}=$ left eye; $\mathrm{pts}=$ points (number); $\mathrm{L} \mathrm{S}=$ low sensitivity. 


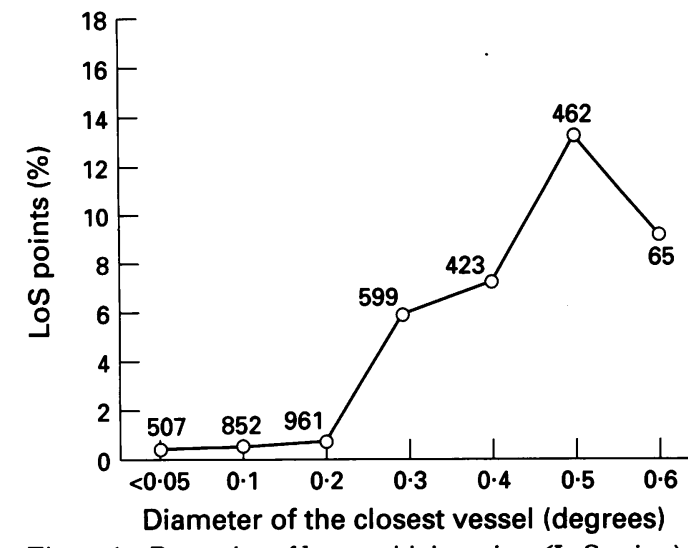

Figure 4 Proportion of low sensitivity points ( $L O S$ points) in relation to the diameter of the closest vessel. Sample sizes are indicated on the graph.

(Fig 4). This proportion appeared to be negligible in the absence of a nearby vessel, or when the closest vessel was less than $0.3^{\circ}$ in diameter. In contrast, when vessels were $0.3^{\circ}$ or larger in diameter, the proportion of LoS points markedly increased, with values ranging from $5.9 \%$ to $13.2 \%$. Increase in the diameter of vessel 1 by only $0 \cdot 1^{\circ}$ was associated with an increase in the proportion of LoS points; the difference was significant when the diameter increased from $0.2^{\circ}$ to $0.3^{\circ}$, or from $0.4^{\circ}$ to $0.5^{\circ}$ ( $p<0.005$ using the $\chi^{2}$ test).

We also evaluated the influence of the diameter of vessel 1 , taking into account that of vessel 2 . It appeared that when vessel 1 was $0 \cdot 1^{\circ}$ or $0 \cdot 2^{\circ}$ in diameter, the influence of vessel 2 was minimal; in contrast, when vessel 1 was $0.3^{\circ}$ or more in diameter, the presence of vessel 2 showed a marked influence (Fig 5 ).

INFLUENCE OF THE DISTANCE FROM VESSEL 1 The influence of the distance from vessel 1 is shown in Figure 6. The proportion of LoS points was negligible when the light spot was separated from the vessel by $0.25^{\circ}$ or more. In contrast, the proportion increased when the tested location was adjacent to vessel 1 , and reached $14.9 \%$ when the light spot was centred on the vessel. This phenomenon occurred when the closest vessels were $0.3^{\circ}$ or more in diameter. When tested locations were centred

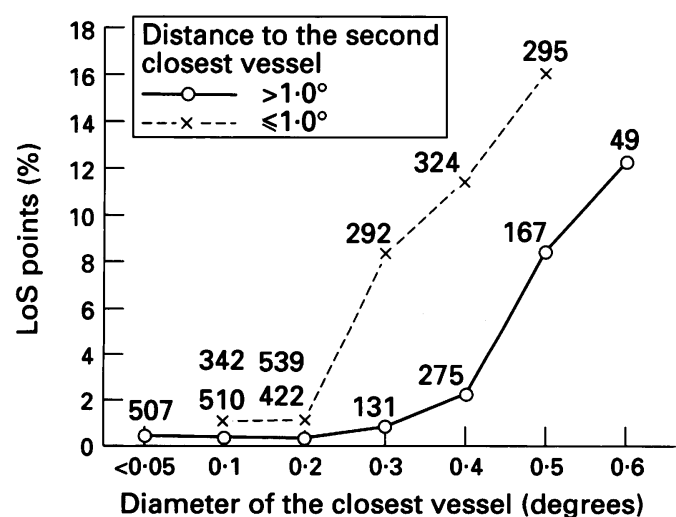

Figure 5 Proportion of low sensitivity points (LoS points) in relation to the diameter of the closest vessel (continuous line: there is no second vessel within a distance of $1 \cdot 0^{\circ}$ from the tested location; interrupted line: a second vessel is present within a distance of $\left.1 \cdot 0^{\circ}\right)$. Sample sizes are indicated on the graph. on these vessels, the proportion of LoS points was markedly increased: it was $12.3 \%$ with vessels $0.3^{\circ}$ in diameter, and reached $40 \%$ with vessels $0.6^{\circ}$ in diameter.

INFLUENCE OF THE DISTANCE FROM VESSEL 2

The distance from vessel 2 was also influential. The proportion of LoS points increased steadily as the distance decreased, starting at $1.5 \%$ when the second vessel was $1 \cdot 25^{\circ}$ distant or more, and reaching $57 \cdot 1 \%$ when the tested location was superimposed on vessel 2 - that is, when the stimulus was projected onto the crossing of two vessels.

\section{INFLUENCE OF THE DISTANCE BETWEEN}

VESSELS 1 AND 2

In addition, when analysing the importance of the distance between vessels 1 and 2 , it appeared that when vessel 1 was either $0.3^{\circ}$ or $0.4^{\circ}$ in diameter, the presence of vessel 2 with a diameter of $0 \cdot 2^{\circ}$ or more was influential only when the distance between the vessels was less than $0.5^{\circ}$ (Figs 7 and 8 ).

\section{RESULTING RULES}

It appears from these results that the following two morphological conditions cause a reduction in sensitivity: (1) points adjacent to a large vessel (that is, distance $<0.25^{\circ}$ and diameter $0.5^{\circ}$ to $\left.0.6^{\circ}\right) ;(2)$ points adjacent $\left(<0.25^{\circ}\right)$ to a moderately large vessel $\left(0.3^{\circ}\right.$ to $0.4^{\circ}$ in diameter) and close $\left(<0.5^{\circ}\right.$ distant $)$ to another vessel at least $0 \cdot 2^{\circ}$ in diameter.

$\chi^{2}$ tests were performed to confirm this supposition. In condition $1, \chi^{2}$ was equal to $314.15(\mathrm{p}<0 \cdot 001)$. Condition 2 was considered only for those points which did not meet condition 1 ; a $\chi^{2}$ test, using only those 3502 points, showed a value of 284.28 $(\mathrm{p}<0.001)$.

These relations were further investigated by calculating positive predictive values (PPV), negative predictive values (NPV), sensitivity, and specificity, first with the data pooled from all tested eyes and then with those of each tested eye separately (Table 1 ).

In the studied areas of the visual field, the proportion of $\operatorname{LoS}$ locations varied from one subject to another and, in one tested individual (subject 2), from one eye to the other. In this subject, the difference in proportion of LoS points found between the eyes was explained by the difference in the morphological characteristics of the patient's vessels, as reflected by the number of locations corresponding to condition 1 or 2 (Table 1 ).

\section{Discussion}

Vessel interference with light projection onto the retina is a cause of spatial variation in light sensitivity. ${ }^{1}$ In our subjects, $3 \cdot 8 \%$ of tested points in the vicinity of the blind spot showed reduced sensitivity under routine conditions of clinical evaluation using the Octopus 2000R perimeter. When a light spot was projected 


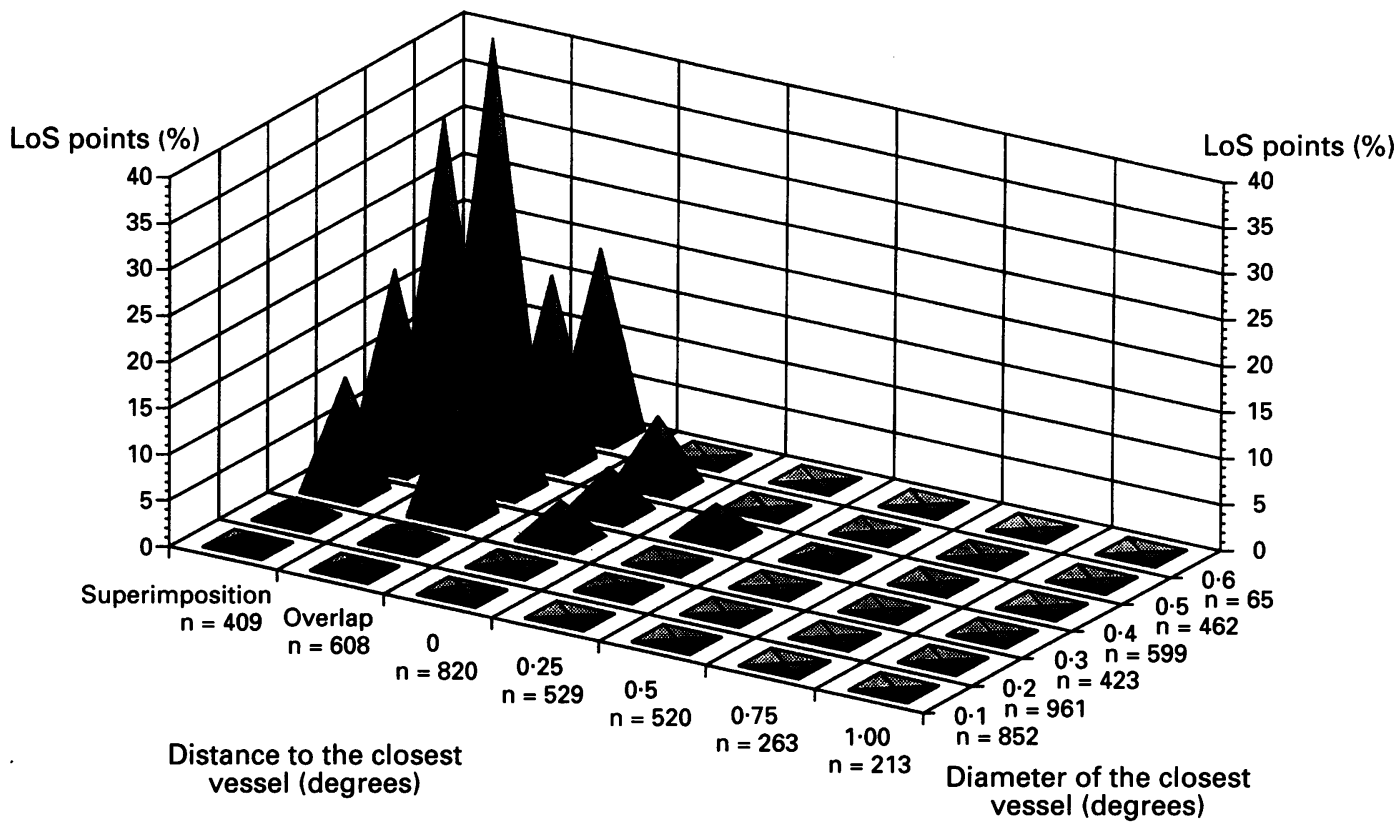

Figure 6 Proportion of low sensitivity points (LoS points) when both the distance and the diameter of the closest vessel are taken into account.

onto the centre of a vessel $0.5^{\circ}$ in diameter, the proportion was much higher - that is, $34 \cdot 6 \%$.

Angioscotomata were extensively studied several decades ago using manual perimetry, ${ }^{1}$ but few studies on this topic used automated perimetry. The use of a spatially adaptive strategy in delineating angioscotomata, with a $0 \cdot 2^{\circ}$ resolution grid and a Goldmann I stimulus size, was evaluated by Haeberlin et al..$^{23}$ Angioscotomata have been found to measure about $0.6^{\circ}$ in width, and usually show a $10 \mathrm{~dB}$ reduction in sensitivity. In an evaluation by Zingirian and colleagues of the pericaecal area using automated perimetry, with tested points at intervals of $3^{\circ}$ horizontally and $7 \cdot 5^{\circ}$ vertically, large variations in sensitivity were observed around the blind spot and were attributed to the presence of large vessels in this area. ${ }^{4}$ Later, angioscotomata were detected by Masukagami et al using fundus photoperimetry in the pericaecal area of both normal individuals and highly myopic patients. ${ }^{5}$ Finally, Zulauf studied the crossing of two large vessels in the retina of one subject. $\mathrm{He}$ reported angioscotomata as deep at $8 \mathrm{~dB}$

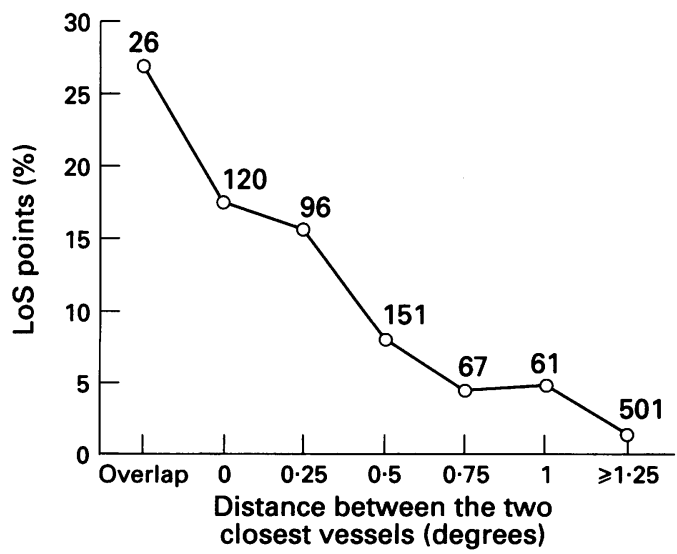

Figure 7 Proportion of low sensitivity points ( $L O S$ points) in relation to the distance between the two closest vessels. Only those points are considered where the diameter of the closest vessel is $0.3^{\circ}$ to $0.4^{\circ}$. using a Goldmann III stimulus, and deeper angioscotomata values using smaller stimuli; in this study, however, visual sensitivity was not correlated with vessel diameter. ${ }^{6}$

To our knowledge, the present investigation is the first quantified evaluation, involving several subjects, of the relations between the morphological features of retinal vessels and corresponding alterations in light sensitivity, under routine conditions of automated perimetry. Our results provide specific indications which may be of both theoretical and practical interest.

From the two conditions that were identified as being related to a reduction in sensitivity, we concluded that reduction in sensitivity to below $26 \mathrm{~dB}$ was related to the masking effect induced either by a retinal vessel of at least $0.5^{\circ}$ in diameter, or by two adjacent retinal vessels totalling at least $0.5^{\circ}$ in width, the closest vessel being at least $0.3^{\circ}$ in diameter. The morphological features of the vessels which we found to be related to the production of angioscotomata are, fortunately, easily recognisable on routine examination of the fundus: the first condition mentioned above involves large vessels, which may or may not be isolated, and the second condition involves two medium sized vessels, which must be adjacent. Evaluation of these rules using positive predictive values, negative predictive values, and statistical sensitivity and specificity supported these definitions.

In the area surrounding the blind spot, the proportion of LoS locations markedly varied from one subject to another. It occasionally varied from one eye to the other in the same individual, as shown with subject 2 (Figs 1 and 2). It is likely that the observed interindividual and interocular differences in the proportion of LoS points were not entirely due to changes in collaboration during the examination, as they were correlated with differences in the proportion of locations included in the conditions associated with a reduction in light sensitivity. 


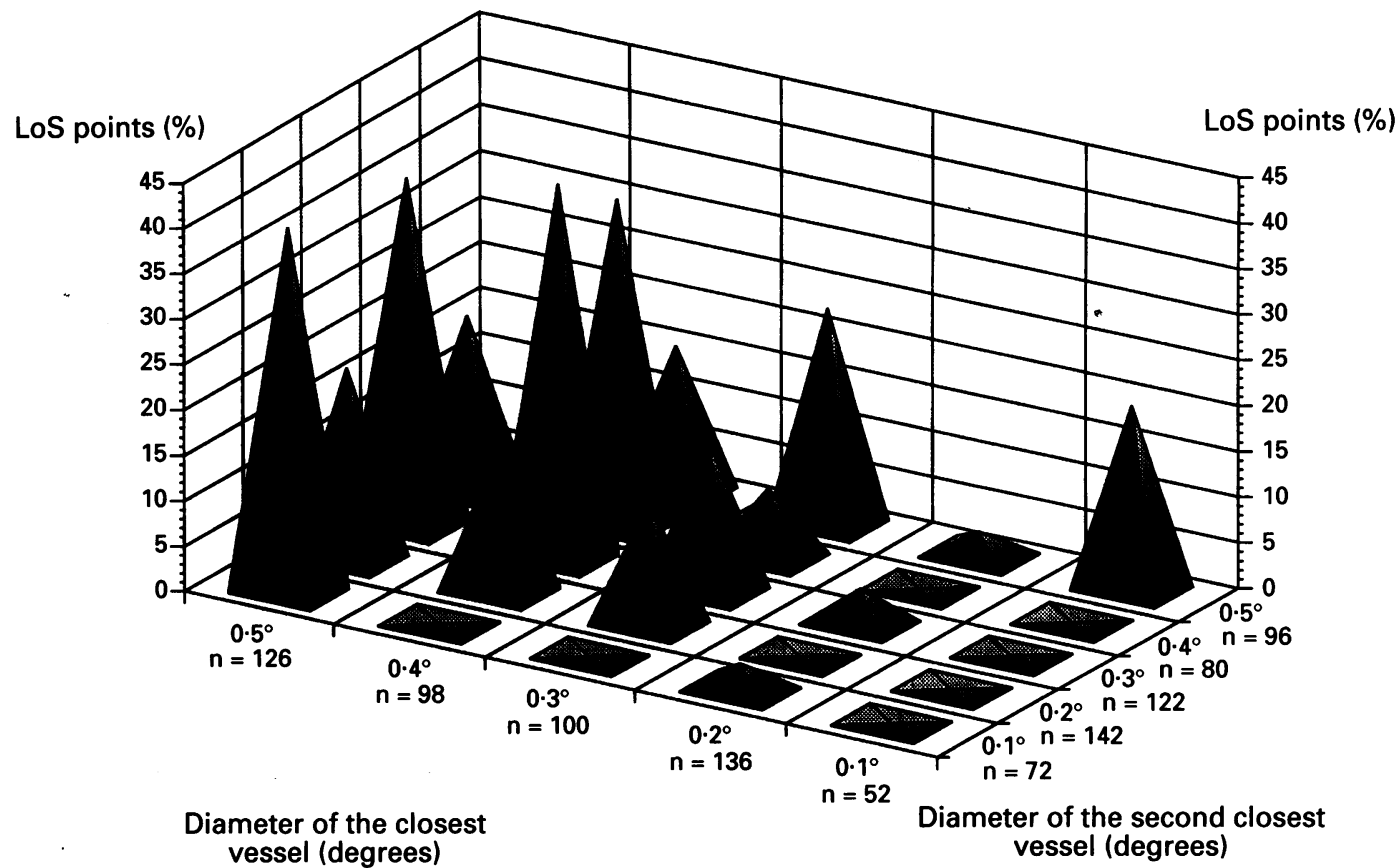

Figure 8 Proportion of low sensitivity points (LoS points) when the diameters of the closest and second closest vessels are taken into account. Only those points are considered which are less than $0 \cdot 25^{\circ}$ from vessel 1 , and where the distance between vessels 1 and 2 is less than $0.5^{\circ}$.

In subject 2, the proportion of LoS points was twice as large in the right eye as in the left eye, and the number of locations satisfying condition 2 was much larger in the right eye.

Analysis of the influence of the diameter of vessel 1 showed that, as a rule, vessel 1 can be categorised into three main groups: (1) small vessels up to $0.2^{\circ}$ in diameter, which are associated with only minimal reduction in sensitivity; (2) medium sized vessels, $0 \cdot 3^{\circ}$ to $0.4^{\circ}$ ) in diameter, which, essentially, are associated with a marked reduction in sensitivity when adjacent to a second vessel of at least medium size; and (3) large vessels, which alone cause a marked reduction in sensitivity (Figs 5 and 8). Interestingly we found that, when the width of vessel 1 was originally either $0.2^{\circ}$ or $0.4^{\circ}$, an increase in the diameter by only $0.1^{\circ}$ resulted in a significant decrease in sensitivity of the neighbouring retina. In such conditions, therefore, $\mathrm{a} 0 \cdot 1^{\circ}$ increase in diameter promoted the involved vessel into the next larger category - that is, from a small vessel to a medium sized vessel, or from a medium sized vessel to a large vessel. In contrast, adding $0 \cdot 1^{\circ}$ to vessels $0.3^{\circ}$ or $0.5^{\circ}$ in diameter did not result in a significant reduction in sensitivity, as the modified vessels remained in the same functional category.

When vessels 1 and 2 were both medium sized - that is, $0.3^{\circ}$ to $0.4^{\circ}$ in diameter, the closest vessel was generally larger than vessel 2 . Although the proximity of vessels 1 and 2 was found to contribute to a reduction in light sensitivity, this observation indicates that perception of light spots was affected more when they were projected in the vicinity of the larger of the two vessels, than when they were projected in the vicinity of the smaller vessel. This might be explained by the fact that, when two medium sized vessels lie adjacent to one another, usually the larger is a vein and the smaller is an artery. The vein, being larger than the artery, constitutes a wider obstacle to the projection of light onto the retina; being darker, it also absorbs more of the projected light. In this study, we were unable to dissociate the effects of the dimension and the nature of the vessel, as veins were usually of larger diameter then arteries.

Considering that the light stimulus used in this study was $0.431^{\circ}$ in diameter (equivalent in size to the Goldmann III stimulus), it appeared that the involved vessel should be at least as wide as the light stimulus in order to induce a significant reduction in retinal sensitivity. Similarly, when the masking effect resulted from the cumulative influences of two neighbouring medium sized vessels, the required total surface was apparently of the same magnitude as that of a single large vessel. Our quantified study corroborated the assumption of Haeberlin $e t a l_{,}^{2}$ that angioscotomata might occur when the diameters of the main retinal vessels near the tested location are roughly the same size as the stimuli projected upon the retina.

Based on our results, it would be inappropriate to analyse further the morphological characteristics of the vessels associated with reduction in light sensitivity, because a number of factors affecting the precision of the measurements obtained in this study cannot be properly quantified. While one may elaborate on the error in measurements which might result from a procedure based on superposing the printed results from the visual field onto a photograph of the fundus, ${ }^{8-11}$ it would be hazardous to evaluate the error resulting from unsteadiness of fixation. ${ }^{12}$

Quantifying the effects of various morphological features of retinal vessels on the occurrence of angioscotomata would have been more accurate using techniques based on 
fundus perimetry. ${ }^{51314}$ However, while such systems undoubtedly permit observations presently unequalled in precision, our study using automated perimetry techniques better illustrates the changes which we regularly observe when performing routine examinations of the visual field, using instruments which are recognised as the most sophisticated for routine clinical evaluation of the visual field. This was precisely the purpose of our study.

The observations made in our study are of practical importance. Firstly, they allow a better understanding of the normality criteria of spatial variations in retinal sensitivity. Secondly, they suggest that, in certain circumstances, alteration in the diameter of a retinal vessel over time might cause fluctuations in the differential light threshold measured in neighbouring parts of the retina. Modifications in vessel diameter are observed in a number of disorders, including obstructive changes in retinal vessels. ${ }^{15}$ They also occur as a normal adaptive response to changes in ambient conditions. Furthermore, there is evidence that autoregulatory systems occasionally result in dramatic retinal vascular changes; thus, in monkeys, for example, it has been shown that hypercapnia causes dilatation of the retinal vessels, with an increase in diameter of up to $50 \% .^{16}$

This suggests that, when the transparent media of the eye are unaltered and the subject collaborates adequately, long term fluctuations in measured retinal sensitivity do not necessarily reflect, as is commonly stated, functional changes in nervous tissues in the visual pathways.

Supported in part by the grant No 32.27842 .89 from the Swiss National Fund for Scientific Research.
1 Dubois-Poulsen A, François P, Tibi A, Magis C. Le Champ Visuel. Topographie normale et pathologique de ses sensibilités. Paris: Masson, 1952: 257-308.

2 Haberlin H, Jenni A, Fankhauser F. Researches on adaptive high resolution programming for automatic perimeter. high resolution programming

3 Haberlin H, Funkhouser A, Fankhauser F. Angioscotoma: preliminary results using the new spatially adaptive program SAPRO. In: Greve EL, Heijl A, eds. Fifth international visual field symposium. The Hague: $\mathrm{Dr} W$ Junk Publishers, 1983: 337-43.

4 Zingirian M, Calabria G, Gandolfo E, Sandini G. The normal pericoecal area. A static method for investigation. In: Greve EL, Heijl A, eds. Fourth international visual field symposium.

5 Masukagami H, Furuno F, Matsuo $\mathrm{H}$. Blind spots of normal and high myopic eyes measured by fundus photoperimetry. In: Greve EL, Heijl A, eds. Seventh interperimetry. In: Greve EL, Heill A, eds. Seventh international visual field symposium, Documenta Ophthalmologica Proceedings Series 49. Dordrecht:
Junk Publishers, 1987: 489-93.

6 Zulauf M. Beitrag zur Angioskotometrie. Stimulusgrösse und Programmwahl. Klin Monatsbl Augenheilkd 1988; 192: 613-8.

7 Safran AB, Mermoud C, Estreicher J, Liebling TM. Evaluation of the blind spot in automated perimetry using a spatially adaptive strategy: optimization of the procedure by means of computerized simulation. In: Mills RP, ed. Perimetry update 1992/93. Amsterdam: Kugler Perimetry update 1992/93.

8 Frisen L. The cartographic deformations of the visual field. Ophthalmologica 1970; 161: 38-54.

9 Kirkham TH, Meyer E. Visual field area on the Goldmann hemispheric perimeter surface. Correction of cartographic errors inherent in perimetry. Curr Eye Res 1981; 1: 93-9.

10 Bek T, Lund-Andersen $H$. Accurate superimposition of perimetry data onto fundus photographs. Acta Ophthalmol 1990; 68: 11-8.

11 Bek T. Localized scotomata and types of vascular occlusion in diabetic retinopathy. Acta Ophthalmol 1991; 69: 11-8.

12 Eizenman M, Trope GE, Fortinsky M, Murphy PH. Stability of fixation in healthy subjects during automated perimetry. Can $₹$ Ophthalmol 1992; 27: 336-40.

13 Timberlake GT, Mainster MA, Webb RH, Hughes GW, Timberlake GT, Mainster MA, Webb RH, Hughes GW,
Trempe CL. Retinal localization of scotomata by scanTrempe CL. Retinal localization of scotomata by scan1982; 22: 91-7.

14 Stuermer J, Schroedel C, Rappl W. Scanning laser ophthalmoscope for static fundus perimetry in glaucomatous nerve-fiber-bundle defects. In: Mills RP, Heijl A, eds Perimetry update 1990/91. Amsterdam: Kugle Publications, 1991: 85-92.

15 Ernest JT. Regulatory mechanisms of the retinal vasculature in health and disease. In: Tso MOM, ed. Retinal disease. Biomedical foundations and clinical management. Philadelphia: Lippincott, 1987: 131-7.

16 Tsacopoulos $\mathrm{M}$, David NJ. The effect of arterial $\mathrm{pCO}_{2}$ on relative retinal blood flow in monkeys. Invest Ophthalmol Vis Sci 1973; 12: 335-47. 